\title{
STEADY STATES OF THE 1D DOI-ONSAGER MODEL IN THE STRONG SHEAR FLOW*
}

\author{
GERALD WARNECKE ${ }^{\dagger}$ AND HUI ZHANG $\ddagger$
}

\begin{abstract}
Here we will investigate the steady states of the Doi-Onsager model in general shear flows. Generally we know that the equilibrium is not the Maxwell-Boltzmann distribution in the shear flow. Here we will give not only the expression of the equilibrium for the 1D Doi-Onsager model in shear flows by rigorous proof, but also a discussion of the critical points of the intensity $\alpha$ and the shear rate $\gamma$ by numerical calculation. The equilibrium of the particles depends on the competition between the parameters $\alpha$ and $\gamma$. When $\alpha$ crosses the first critical point $\alpha_{1} \approx 4.083$ from a smaller value, the number of steady states will change from one to three through two. At the same time the shear rate $\gamma$ depends on $\alpha$. When $\alpha$ crosses the second critical point $\alpha_{2} \approx 5.125$ from smaller values, the number of steady states will change from three to one through two for some related $\gamma$. It can be found that some of the equilibria are stable with respect to the energy functional. These results are partly consistent with the work of Marrucci and Maffettone in [20], in which formal analysis was given.
\end{abstract}

Key words. Steady states, strong shear flow, Doi-Onsager model, critical points.

AMS subject classifications. 82B27, 35Q35.

\section{Introduction}

It is well-known that the Doi model can describe the dynamical states in dilute polymeric fluids. We consider the behaviour of chain molecules or rod particles. Important effects were found in some numerical simulations, e.g., [9, 10, 11, 14, 19, 20], and theoretical analysis, e.g., $[1,2,3,26]$. In the simplest case of no flow (velocity $u=0)$, it is also called Doi-Onsager model $[17,23,25]$. It can describe phase transition phenomena though it is very simple. Therefore, this model has recently attracted considerable attention $[4,5,6,14]$, especially in the mathematics community $[1,2,3$, $7,8,12,13,16,17,18,23,24]$. At first, Onsager [22] showed in 1949 that the steady state is the prolate phase when the intensity among the particles is strong. The prolate phase consists of aligned particles. He derived this result via a variational approach under the assumption that the equilibrium was axially symmetric and possessed some explicit expression for the Onsager interaction potential between molecules. This name was attributed to the potential by later authors. In 1959 Maier and Saupe [21] investigated the nematic phase for a simpler potential among the molecules, which is now called Maier-Saupe potential, e.g., [4, 5]. In 2005, a rigorous proof of the Onsager assumption for the Maier-Saupe potential was given in $[17,8,23]$. Meanwhile explicit expressions for all equilibria were shown in $[3,17]$. The phase transition phenomenon was proved in the investigation of stability of these equilibria [24]. In [24], many steady states were found in the weak flow for the Doi model, such as flow-aligning, tumbling, log-rolling, and kayaking. Concerning the asymptotic states and the properties of the Doi-Onsager model without flow, Constantin et al. [1,2] gave many very interesting

\footnotetext{
${ }^{*}$ Received: February 20, 2009; Accepted (in revised version): September 12, 2009.

${ }^{\dagger}$ Institute for Analysis and Numerics, Otto-von-Guericke-University Magdeburg, Universitätsplatz 2, D-39106 Magdeburg, Germany (warnecke@ovgu.de).

$\ddagger$ Corresponding author. School of Mathematical Sciences, Beijing Normal University, Key Laboratory of Mathematics and Complex Systems, MCE, Beijing, 100875, P.R. China (hzhang@bnu.edu.cn). This work is partially supported by an Alexander von Humboldt Fellowship at Otto-von-GuerickeUniversity Magdeburg, the key basic research project of the Ministry of Chinese Education 107016 and the state key basic research project of China 2005CB321704.
} 
results. Here we will investigate the equilibrium of the Doi-Onsager model in the strong shear flow while we restrict ourselves to rodlike particles on the circle.

The mathematical model we adopt is the Doi-Onsager equation on the circle for shear flow

$$
\psi_{t}=D_{r}\left[\psi_{\theta \theta}+\left(\psi U_{\theta}\right)_{\theta}\right]+\gamma\left(\psi \sin ^{2} \theta\right)_{\theta},
$$

where $\psi(\theta, t)$ denotes the orientational probability distribution function (PDF) for rod-like, rigid molecules. The constant $D_{r}$ is the averaged rotational diffusivity, which will be set to 1 . The parameter $\gamma$ is the shear rate. The function $U$ is the mean-field interaction potential, which here is considered to be the Maier-Saupe potential [21] given as

$$
U=\alpha \int_{0}^{2 \pi} \sin ^{2}\left(\theta-\theta^{\prime}\right) \psi\left(\theta^{\prime}, t\right) d \theta^{\prime}
$$

The parameter $\alpha$ describes the interaction intensity among the rod particles. Furthermore, we know that $\psi(\theta, t)$ is $2 \pi$ periodic in $\theta$. We are seeking solutions that satisfy $\psi \geq 0$ and the normalization condition

$$
\int_{0}^{2 \pi} \psi(\theta, t) d \theta=1 .
$$

When there is no flow, all equilibria of equation (1.1) were given in [3, 16]. It was shown that if the interaction intensity parameter $\alpha$ is less than 4 , the constant solution is the only possible steady state solution, which describes the isotropic phase. If $\alpha$ is larger than 4, then there is a new solution that corresponds to the nematic phase. All other non-constant solutions are obtained from this solution by translation. It was also proved that the nematic solutions have period $\pi$ instead of $2 \pi$, which signifies the fact that the rods are symmetric, i.e., they have orientations but no directions. Later in [17], a new simple approach to reprove these results was found. Moreover, an explicit expression for all the solutions was given in [17], which is

THEOREM 1.1. [17] The number of stationary solutions to (1.1)-(1.3) with $\gamma=0$ depends on whether the intensity crosses the critical value $\alpha=4$. More precisely,

(i) If $\alpha \leq 4$, then the only stationary solution is the constant $f=1 / 2 \pi$.

(ii) If $\alpha>4$, then besides the constant solution $f=1 / 2 \pi$, all other stationary solutions are given by

$$
f(\theta)=\frac{1}{\tilde{Z}} e^{-\eta^{*} \cos 2\left(\theta-\theta_{0}\right)},
$$

where $\tilde{Z}=\int_{0}^{2 \pi} e^{-\eta^{*} \cos 2 \theta} d \theta$ and $\theta_{0}$ is arbitrary. $\eta^{*}>\frac{\alpha}{2} \sqrt{1-4 / \alpha}$ is uniquely determined by the equation

$$
\frac{\int_{0}^{2 \pi} \cos 2 \theta e^{-\eta^{*} \cos 2 \theta} d \theta}{\int_{0}^{2 \pi} e^{-\eta^{*} \cos 2 \theta} d \theta}+\frac{2 \eta^{*}}{\alpha}=0 .
$$

There are complete theoretical results for the full Doi model on a sphere given in [17]. All equilibrium solutions are necessarily uniaxially symmetric, see [17, 23, 8]. The number of equilibrium states and their qualitative behavior for the Doi-Onsager 
equation hinge on whether the intensity $\alpha$ crosses two critical values [17]. It is also shown that there is a phase transition when $\alpha$ changes. Additionally, we can see the hysteresis phenomenon when the intensity $\alpha$ changes from small to large and from large to small in [24].

In this paper we will determine all equilibria of the Doi equation on the circle under strong shear flows. We obtain the result that there is no isotropic phase and all equilibria are in the nematic phase.

THEOREM 1.2. The PDF $\psi$ of the equilibria of (1.1)-(1.3) possesses the form

$$
\psi(\theta)=\frac{1}{Z}[1+b(\theta)] e^{-a(\theta)},
$$

where

$$
\begin{aligned}
& a(\theta)=\frac{\alpha}{2}+\eta \cos 2\left(\theta-\theta_{0}\right)+\frac{\gamma \theta}{2}, \\
& b(\theta)=\left(e^{\gamma \pi}-1\right) \frac{\int_{0}^{\theta} e^{a(\tau)} d \tau}{\int_{0}^{2 \pi} e^{a(\tau)} d \tau},
\end{aligned}
$$

and $Z$ is the normalization constant, given by

$$
Z=\int_{0}^{2 \pi}[1+b(\theta)] e^{-a(\theta)} d \theta .
$$

Furthermore, the parameters $\eta$ and $\theta_{0}$ are determined by the intensity $\alpha$ and the shear rate $\gamma$ through the relations

$$
\begin{aligned}
& \frac{\int_{0}^{2 \pi} \cos 2\left(\theta-\theta_{0}\right)[1+b(\theta)] e^{-a(\theta)} d \theta}{\int_{0}^{2 \pi}[1+b(\theta)] e^{-a(\theta)} d \theta}+\frac{2 \eta}{\alpha}+\frac{\gamma}{2 \alpha} \sin 2 \theta_{0}=0, \\
& \frac{\int_{0}^{2 \pi} \sin 2\left(\theta-\theta_{0}\right)[1+b(\theta)] e^{-a(\theta)} d \theta}{\int_{0}^{2 \pi}[1+b(\theta)] e^{-a(\theta)} d \theta}+\frac{\gamma}{2 \alpha} \cos 2 \theta_{0}=0
\end{aligned}
$$

Comparing the expression (1.6) with (1.4), we can formally see how far the equilibrium of the 1D Doi model in the general shear flow is from the Boltzmann distribution (1.4) without shear flow. Let us change the form of (1.6) slightly as follows:

$$
\psi(\theta)=\frac{\tilde{Z}}{Z_{1}} e^{-\frac{\gamma \theta}{2}} \frac{1}{\tilde{Z}} e^{-\eta \cos 2\left(\theta-\theta_{0}\right)}+\frac{1}{Z} b(\theta) e^{-a(\theta)},
$$

where $Z_{1}=\int_{0}^{2 \pi} e^{-\left(\frac{\gamma \theta}{2}+\eta \cos 2\left(\theta-\theta_{0}\right)\right)} d \theta$ and we have deleted a term $e^{-\alpha / 2}$. Here the first term is a dilation of $\frac{\tilde{Z}}{Z_{1}} e^{-\frac{\gamma \theta}{2}}$ of the Boltzmann distribution (1.4) due to the shear flow, and we can take the second term as the additional perturbation of the Boltzmann distribution (1.4).

Unfortunately, here we cannot theoretically analyze equations (1.10) and (1.11) to find the critical points of the intensity parameter $\alpha$ and the shear rate $\gamma$. However, by numerical calculations for (1.10) and (1.11), it can be shown that the critical points of the intensity $\alpha$ and the shear rate $\gamma$ are approximate. Precisely, we find the following results:

(a) When $0<\alpha<\alpha_{1}\left(\alpha_{1} \approx 4.083\right)$, there is only one pair of $\left(\eta, \theta_{0}\right)$ to satisfy $(1.10)$ and (1.11). This implies that the equilibrium is uniquely determined by the intensity 
$\alpha$ and the shear rate $\gamma$ when $\alpha$ is less than the first critical point $\alpha_{1}$ and $\gamma$ is any given positive constant. Moreover, for $\alpha \in] 0, \alpha_{1}\left[\right.$ fixed, the solution $\left(\eta, \theta_{0}\right)$ depends on the shear rate $\gamma$ and $\theta_{0}$ is not a monotone function of $\gamma$. There is a critical point for $\gamma$ such that $\theta_{0}$ attains a minimum; for example, $\gamma=3.5$ is the critical point for $\alpha=1.0$ (figure 2.7). We can see that $\eta$ is a monotone increasing function of $\gamma$ for fixed $\alpha$ from Tables 2.1, 2.2 and 2.3 .

(b) When $\alpha_{1}<\alpha<\alpha_{2}\left(\alpha_{2} \approx 5.125\right)$, there are possibly many pairs of solutions $\left(\eta, \theta_{0}\right)$ to satisfy $(1.10)$ and (1.11) for some $\gamma$. The number of solutions depends on the choice of $\gamma$. It shows that the equilibrium of the particles in a polymeric fluid is possible for many steady states, but some steady states are not stable. For example, we can see that $\left(\eta, \theta_{0}\right)=(0.1333,0.8374),(0.967,1.728),(1.0596,1.935)$ are solutions of $(1.10)$ and (1.11) for $\gamma=0.01$ and $\alpha=4.5$ from Table 2.2. But the solution $(0.1333,0.8374)$ is unstable with respect to the energy functional, while for $\alpha=4.5$ and $\gamma=0.1685$, there are two stable solutions $\left(\eta, \theta_{0}\right)=(0.6,1.025),(1.1346,1.9092)$. But when $\alpha=4.5$ and $\gamma>0.6$, there is only one solution. Moreover, it can be found that $\gamma$ depends on $\alpha$ for which there are multiple steady states.

(c) When $\alpha>\alpha_{2}$, there is only one solution $\left(\eta, \theta_{0}\right)$ which satisfies (1.10) and (1.11). This implies that the steady state of the molecules in the flow is also uniquely determined by the interaction intensity $\alpha$ and shear rate $\gamma$. Moreover, for $\alpha \in] \alpha_{2}, \infty[$ fixed, $\theta_{0}$ and $\eta$ are increasing functions of the shear rate $\gamma$, e.g., from (figure 2.10), we can see the profile of $\theta_{0}(\gamma)$ for $\alpha=8.0$.

Here we especially point out that these results are partly consistent with the work of Marrucci and Maffettone in [20]. They formally analyzed the explicit expression for PDF $\psi=C e^{-g(\phi)}\left(\int_{0}^{\phi} e^{-g(x)} d x+K\right)$ with $g(\phi)=-a \cos 2 \phi+G\left(\phi-\frac{1}{2} \sin (2 \alpha+2 \phi)\right)$ and $a=U \int_{0}^{\pi} \psi(\phi) \cos 2 \phi d \phi$ by introducing $\theta=\phi+\beta$, where $\beta$ is the angular shift $(-\pi / 4 \leq \beta \leq \pi / 4)$. The latter is chosen so as to obtain

$$
\int_{0}^{\pi} \psi(\phi) \sin 2 \phi d \phi=0 .
$$

In fact, the parameter $G$ is $\frac{1}{2} \gamma$, and $U$ is about $\frac{1}{2} \alpha$ in our paper since we use the different parameters with a different meaning. Then the result about the critical point of $\alpha$ and $\gamma$ was obtained by numerical calculation to (1.12) for given parameters $a$ and $\beta$ in [20]. It is stated in [20] that:

the solution is not always found, however. We find one (and only one) solution throughout the range of $G$ values explored $\left(0<G<10^{3}\right)$ only for values of $U$ less than ca. 2.41, thus encompassing the whole isotropic range $(0 \leq U \leq 2)$, as well as a small segment of the nematic range $(2<U<2.41)$. Conversely, for $U>2.41$, a lower bound for $G$ is found, call $G_{\text {critical }}$, below which the stationary solution disappears. We infer that, for $G<G_{\text {critical }}$, the system switches to the tumbling situation. $G_{\text {critical }}$ appears to grow steadily with increasing $U$.

Their result was partially correct in virtue of the formal solution. Here we rigorously deduce the form of the solution, and we definitely find that the isotropic solution $\psi=\frac{1}{2 \pi}$ does not satisfy the steady state equation (2.2). So it is obvious that there is no isotropic phase for any positive values of the parameters $\alpha$ and $\gamma$. Furthermore, when $\alpha$ is larger than ca. 5.125, the unique stationary state is the nematic phase for any values of the shear rate $\gamma$. This is also consistent with the result for the case of no flow, see $[3,7,16,17,20]$, which states that the unique stationary state is the nematic phase for $\alpha>4$. In our result we have not found the tumbling situation, which was 
reported in [20], since it is not stable stationary state, . In this work we report that there are two stable stationary states for $\alpha \in] 4.083,5.125[$ and some shear rate $\gamma$. It is possibly due to the presence of wagging state for the time dynamic system. The boundary states of the wagging case would be the stable steady states we found.

In addition, there is a paper [15], in which the weak shear case was investigated for the dynamic model (1.1) with a variety of moment closure approaches. The authors obtained the weak-shear solvability conditions for persistence of equilibria due to the shear-perturbed approach, nearly isotropic branch (using bifurcation software that gives full phase diagram whose regions are separated by bifurcations). These phase diagrams and all bifurcations were compared with the work [21]. Here we directly investigate the steady state equation of the model (1.1) without any closure approach. We also claim that there are one or two stable steady states for some intensity parameter $\alpha$ and the shear rate $\gamma$, which are consistent with the diagram of mesoscopic models in [15].

This paper is organized as follows. Section 2 is devoted to the analysis of the expression and some properties of the equilibrium states in the general shear flow. Meanwhile, we will discuss the critical points of the intensity parameter $\alpha$ and the shear rate $\gamma$, in which the number of the equilibrium changes. In section 3 the stability of multiple solutions was discussed by calculating the energy functional. Then conclusions are drawn in the final section.

\section{Steady states in the shear flow}

In this section we will explicitly investigate the expression of the steady states of equation (1.1) in the shear flow. It follows from (1.2) that

$$
U_{\theta}=\alpha \int_{0}^{2 \pi} \sin 2\left(\theta-\theta^{\prime}\right) \psi\left(\theta^{\prime}, t\right) d \theta^{\prime} .
$$

Furthermore,

$$
\begin{aligned}
U_{\theta \theta} & =2 \alpha \int_{0}^{2 \pi} \cos 2\left(\theta-\theta^{\prime}\right) \psi\left(\theta^{\prime}, t\right) d \theta^{\prime} \\
& =2 \alpha \int_{0}^{2 \pi}\left[1-2 \sin ^{2}\left(\theta-\theta^{\prime}\right)\right] \psi\left(\theta^{\prime}, t\right) d \theta^{\prime} \\
& =2 \alpha-4 U,
\end{aligned}
$$

where (1.3) has been used. Therefore, we obtain a decoupled equation for $\mathrm{U}$

$$
U_{\theta \theta}+4 U=2 \alpha .
$$

Its general solution is

$$
U=\frac{\alpha}{2}+s_{1} \cos 2 \theta+s_{2} \sin 2 \theta
$$

where $s_{1}, s_{2}$ are two parameters.

We consider the steady states, which satisfy

$$
0=\psi_{\theta \theta}+\left(\psi U_{\theta}\right)_{\theta}+\gamma\left(\psi \sin ^{2} \theta\right)_{\theta}
$$

and (2.1). Integration of (2.2) from 0 to $\theta$ yields

$$
\psi_{\theta}+\psi U_{\theta}+\gamma \psi \sin ^{2} \theta=C,
$$


where $C$ is a constant that is independent of $\theta$. If the shear rate $\gamma=0$, we can easily prove that $C \equiv 0$ in the above equation similar to $[16,17]$. Thus the distribution is the Boltzmann distribution $\psi=\frac{1}{Z} e^{-U}$, where $Z$ is the normalization. However we have $C \neq 0$ when $\gamma \neq 0$. In the following we want to determine $C$ in (2.3).

Integration of (2.3) from 0 to $2 \pi$ yields

$$
\int_{0}^{2 \pi} \psi_{\theta} d \theta+\int_{0}^{2 \pi} \psi U_{\theta} d \theta+\int_{0}^{2 \pi} \gamma \psi \sin ^{2} \theta d \theta=2 \pi C .
$$

The first term in (2.4) is zero since $\psi$ is a periodic function of period $2 \pi$. We denote the second term by $G$. Then

$$
\begin{aligned}
G & =\int_{0}^{2 \pi} \psi U_{\theta} d \theta \\
& =\alpha \int_{0}^{2 \pi} \int_{0}^{2 \pi} \sin 2\left(\theta-\theta^{\prime}\right) \psi(\theta) \psi\left(\theta^{\prime}\right) d \theta^{\prime} d \theta \\
& =\alpha \int_{0}^{2 \pi} \int_{0}^{2 \pi}\left(\sin 2 \theta \cos 2 \theta^{\prime}-\cos 2 \theta \sin 2 \theta^{\prime}\right) \psi(\theta) \psi\left(\theta^{\prime}\right) d \theta^{\prime} d \theta \\
& =0 .
\end{aligned}
$$

The last equality is easily seen by writing the difference as two separate integrals and interchanging the variables in one of them. So (2.4) implies

$$
\int_{0}^{2 \pi} \gamma \psi(\theta) \sin ^{2} \theta d \theta=2 \pi C
$$

Thus, we can obtain

$$
C=\frac{\gamma}{2 \pi} \int_{0}^{2 \pi} \sin ^{2} \theta \psi(\theta) d \theta>0
$$

Now we introduce $a^{\prime}(\theta)=U_{\theta}+\gamma \sin ^{2} \theta$, then

$$
\begin{aligned}
a(\theta) & =U+\gamma\left(\frac{\theta}{2}-\frac{1}{4} \sin 2 \theta\right) \\
& =\frac{\alpha}{2}+s_{1} \cos 2 \theta+s_{2} \sin 2 \theta+\gamma\left(\frac{\theta}{2}-\frac{1}{4} \sin 2 \theta\right) \\
& =\frac{\alpha}{2}+\eta \cos 2\left(\theta-\theta_{0}\right)+\frac{\gamma \theta}{2},
\end{aligned}
$$

where $\eta$ and $\theta_{0}$ are two new parameters replacing $s_{1}, s_{2}$ which satisfy

$$
\eta^{2}=s_{1}^{2}+\left(s_{2}-\gamma / 4\right)^{2}, \quad \tan 2 \theta_{0}=\left(s_{2}-\gamma / 4\right) / s_{1} .
$$

Without the loss of the generality we assume here that $\eta>0$. For $\eta<0$ we can modify the definition of $\theta_{0}$ to $\theta_{0}+\pi / 2$ to give the same form of $a(\theta)$. Then from $(2.3)$, we can obtain

$$
\left(\psi e^{a(\theta)}\right)_{\theta}=C e^{a(\theta)} .
$$

Integration of (2.7) from 0 to $\theta$ yields

$$
\psi(\theta) e^{a(\theta)}=K+C \int_{0}^{\theta} e^{a(\tau)} d \tau
$$


where $K$ is a constant. Therefore, we obtain that the distribution function is

$$
\psi(\theta)=K e^{-a(\theta)}+C e^{-a(\theta)} \int_{0}^{\theta} e^{a(\tau)} d \tau,
$$

where $a(\theta)$ and $C$ are given by (2.6) and (2.5), respectively. Here we can see that there are five parameters $K, \alpha, \gamma, \eta, \theta_{0}$ in the expression (2.8). However they are not completely independent. They certainly satisfy some relations as follows:

(1) The distribution function $\psi(\theta)$ satisfies the normalization (1.3). So we have

$$
K \int_{0}^{2 \pi} e^{-a(\theta)} d \theta+C \int_{0}^{2 \pi} e^{-a(\theta)} \int_{0}^{\theta} e^{a(\tau)} d \tau d \theta=1 .
$$

(2) The function $\psi(\theta)$ is periodic, i.e., $\psi(2 \pi)=\psi(0)$. From (2.8) and (2.6), we obtain

$$
\begin{aligned}
& \psi(2 \pi)=K e^{-a(2 \pi)}+C e^{-a(2 \pi)} \int_{0}^{2 \pi} e^{a(\tau)} d \tau, \quad \psi(0)=K e^{-a(0)}, \\
& a(2 \pi)=\frac{\alpha}{2}+\eta \cos 2 \theta_{0}+\gamma \pi=a(0)+\gamma \pi
\end{aligned}
$$

Thus, we have from $\psi(2 \pi)=\psi(0)$ the relation

$$
K\left[e^{\gamma \pi}-1\right]=C \int_{0}^{2 \pi} e^{a(\tau)} d \tau .
$$

From the relation (2.10) we can see that $K$ has the same sign as the shear rate $\gamma$ because $C>0$ and $\int_{0}^{2 \pi} e^{a(\tau)} d \tau>0$.

(3) On the other hand, from (2.1) and (2.6)

$$
\begin{aligned}
U & =\frac{\alpha}{2}+s_{1} \cos 2 \theta+s_{2} \sin 2 \theta \\
& =\frac{\alpha}{2}+\eta \cos 2\left(\theta-\theta_{0}\right)+\frac{\gamma}{4} \sin 2 \theta
\end{aligned}
$$

Invoking (1.2) we have

$$
\frac{\alpha}{2}+\eta \cos 2\left(\theta-\theta_{0}\right)+\frac{\gamma}{4} \sin 2 \theta=\alpha \int_{0}^{2 \pi} \sin ^{2}\left(\theta-\theta^{\prime}\right) \psi\left(\theta^{\prime}\right) d \theta .
$$

From the above relations (2.9)-(2.12) for the parameters $K, \alpha, \gamma, \eta, \theta_{0}$, we can now conclude the result of Theorem 1.2.

The proof of Theorem 1.2.

Proof. From (2.10) we obtain

$$
C=K \frac{e^{\gamma \pi}-1}{\int_{0}^{2 \pi} e^{a(\tau)} d \tau}
$$

We insert this into (2.8) to obtain

$$
\psi(\theta)=K\left[1+\frac{e^{\gamma \pi}-1}{\int_{0}^{2 \pi} e^{a(\tau)} d \tau} \int_{0}^{\theta} e^{a(\tau)} d \tau\right] e^{-a(\theta)} .
$$


Now the definition $Z=1 / K, b(\theta)$ given by (1.8), and $a(\theta)$ already defined in $(2.6)$ gives the form (1.6) of the solution. The formula (1.9) for $Z$ is defined by (2.9) using (2.10) again. Finally we insert the solution (1.6) into (2.12) to obtain

$$
\frac{\int_{0}^{2 \pi} \sin ^{2}\left(\theta-\theta^{\prime}\right)\left[1+b\left(\theta^{\prime}\right)\right] e^{-a\left(\theta^{\prime}\right)} d \theta^{\prime}}{Z}=\frac{1}{2}+\frac{\eta}{\alpha} \cos 2\left(\theta-\theta_{0}\right)+\frac{\gamma}{4 \alpha} \sin 2 \theta .
$$

Now combining (2.13) with (1.9) for the nonlocal term $Z$ we can obtain

$$
\begin{aligned}
& \frac{\int_{0}^{2 \pi} \sin ^{2}\left(\theta-\theta^{\prime}\right)\left[1+b\left(\theta^{\prime}\right)\right] e^{-a\left(\theta^{\prime}\right)} d \theta^{\prime}}{\int_{0}^{2 \pi}[1+b(\theta)] e^{-a(\theta)} d \theta}=\frac{1}{2}+\frac{\eta}{\alpha} \cos 2\left(\theta-\theta_{0}\right)+\frac{\gamma}{4 \alpha} \sin 2 \theta \\
= & \frac{1}{2}+\left[\frac{\eta}{\alpha}+\frac{\gamma}{4 \alpha} \sin 2 \theta_{0}\right] \cos 2\left(\theta-\theta_{0}\right)+\frac{\gamma}{4 \alpha} \cos 2 \theta_{0} \sin 2\left(\theta-\theta_{0}\right) .
\end{aligned}
$$

Since

$$
\begin{aligned}
\sin ^{2}\left(\theta-\theta^{\prime}\right) & =\frac{1}{2}\left[1-\cos 2\left(\theta-\theta^{\prime}\right)\right]=\frac{1}{2}-\frac{1}{2} \cos \left[2\left(\theta-\theta_{0}\right)+2\left(\theta_{0}-\theta^{\prime}\right)\right] \\
& =\frac{1}{2}-\frac{1}{2}\left[\cos 2\left(\theta-\theta_{0}\right) \cos 2\left(\theta_{0}-\theta^{\prime}\right)-\sin 2\left(\theta-\theta_{0}\right) \sin 2\left(\theta_{0}-\theta^{\prime}\right)\right] \\
& =\frac{1}{2}-\frac{1}{2}\left[\cos 2\left(\theta-\theta_{0}\right) \cos 2\left(\theta^{\prime}-\theta_{0}\right)+\sin 2\left(\theta-\theta_{0}\right) \sin 2\left(\theta^{\prime}-\theta_{0}\right)\right],
\end{aligned}
$$

we have that the left side of (2.14) is equal to

$$
\begin{aligned}
\frac{1}{2} & -\frac{1}{2} \frac{\int_{0}^{2 \pi} \cos 2\left(\theta^{\prime}-\theta_{0}\right)\left[1+b\left(\theta^{\prime}\right)\right] e^{-a\left(\theta^{\prime}\right)} d \theta^{\prime}}{\int_{0}^{2 \pi}[1+b(\theta)] e^{-a(\theta)} d \theta} \cos 2\left(\theta-\theta_{0}\right) \\
- & \frac{1}{2} \frac{\int_{0}^{2 \pi} \sin 2\left(\theta^{\prime}-\theta_{0}\right)\left[1+b\left(\theta^{\prime}\right)\right] e^{-a\left(\theta^{\prime}\right)} d \theta^{\prime}}{\int_{0}^{2 \pi}[1+b(\theta)] e^{-a(\theta)} d \theta} \sin 2\left(\theta-\theta_{0}\right) .
\end{aligned}
$$

Using the linear independence of $\cos 2\left(\theta-\theta_{0}\right)$ and $\sin 2\left(\theta-\theta_{0}\right)$ gives the two relations (1.10) and (1.11).

From the expression (1.6) we can see that the distribution function $\psi$ is an approximate Boltzmann distribution (1.4) in [17] when the shear rate $\gamma$ is sufficiently small since

$$
e^{\gamma \pi}-1 \approx 0 \text { as } \gamma \ll 1
$$

and

$$
\frac{\alpha}{2}+\eta \cos 2\left(\theta-\theta_{0}\right)+\frac{\gamma \theta}{2} \approx \frac{\alpha}{2}+\eta \cos 2\left(\theta-\theta_{0}\right) \text { as } \gamma \ll 1
$$

Next we will prove the periodicity property of this distribution function in the strong shear flow similar to the equilibrium of the distribution function in no flow $[16,17]$.

Proposition 2.1. The solution $\psi(\theta)$ defined by (1.6) is periodic with period $\pi$.

Proof. By using (1.7), we can obtain

$$
\begin{aligned}
\int_{0}^{\theta+\pi} e^{a(\tau)} d \tau & =\left(\int_{0}^{\pi}+\int_{\pi}^{\theta+\pi}\right) e^{a(\tau)} d \tau \\
& =\int_{0}^{\pi} e^{a(\tau)} d \tau+e^{\frac{\gamma \pi}{2}} \int_{0}^{\theta} e^{a(\tau)} d \tau
\end{aligned}
$$


Thus, in virtue of (1.6)-(1.8)

$$
\begin{aligned}
\psi(\theta+\pi) & =\frac{1}{Z}[1+b(\theta+\pi)] e^{-a(\theta+\pi)} \\
& =\frac{1}{Z}\left[1+\frac{e^{\gamma \pi}-1}{\int_{0}^{2 \pi} e^{a(\tau)} d \tau}\left(\int_{0}^{\pi} e^{a(\tau)} d \tau+e^{\frac{\gamma \pi}{2}} \int_{0}^{\theta} e^{a(\tau)} d \tau\right)\right] e^{-a(\theta)-\frac{\gamma \pi}{2}} \\
& =\frac{1}{Z}\left[1+\frac{e^{\gamma \pi}-1}{\int_{0}^{2 \pi} e^{a(\tau)} d \tau} \int_{0}^{\pi} e^{a(\tau)} d \tau\right] e^{-a(\theta)-\frac{\gamma \pi}{2}}+\frac{1}{Z} \frac{e^{\gamma \pi}-1}{\int_{0}^{2 \pi} e^{a(\tau)} d \tau} \int_{0}^{\theta} e^{a(\tau)} d \tau e^{-a(\theta)} \\
& =\frac{1}{Z}[1+b(\pi)] e^{-\frac{\gamma \pi}{2}} e^{-a(\theta)}+\frac{1}{Z} b(\theta) e^{-a(\theta)} \\
& =\frac{1}{Z}[1+b(\pi)] e^{-\frac{\gamma \pi}{2}} e^{-a(\theta)}+\psi(\theta)-\frac{1}{Z} e^{-a(\theta)}
\end{aligned}
$$

If we want to prove $\psi(\theta+\pi)=\psi(\theta)$, we have to verify

$$
[1+b(\pi)] e^{-\frac{\gamma \pi}{2}}=1 .
$$

That is, by using (1.8) again,

$$
\left(e^{\gamma \pi}-1\right) \int_{0}^{\pi} e^{a(\tau)} d \tau=\left(e^{\gamma \pi / 2}-1\right) \int_{0}^{2 \pi} e^{a(\tau)} d \tau
$$

In fact, from (1.7) we know that $a(\pi+\theta)=a(\theta)+\frac{\gamma \pi}{2}$. Thus

$$
\begin{aligned}
\int_{0}^{2 \pi} e^{a(\tau)} d \tau & =\left(\int_{0}^{\pi}+\int_{\pi}^{2 \pi}\right) e^{a(\tau)} d \tau \\
& =\int_{0}^{\pi} e^{a(\tau)} d \tau+\int_{0}^{\pi} e^{a(\pi+\tau)} d \tau \\
& =\left(1+e^{\gamma \pi / 2}\right) \int_{0}^{\pi} e^{a(\tau)} d \tau
\end{aligned}
$$

Multiplying by $\left(e^{\gamma \pi / 2}-1\right)$ implies $(2.16)$ and completes the proof.

From this assertion, we know that the rods are symmetric, i.e they have orientations but no directions. This is similar to the result in $[16,17]$.

In the following we will determine the critical points of $\alpha$ and $\gamma$ numerically. Set

$$
\begin{aligned}
& B\left(\alpha, \eta, \gamma, \theta_{0}\right)=\frac{\int_{0}^{2 \pi} \cos 2\left(\theta-\theta_{0}\right)[1+b(\theta)] e^{-a(\theta)} d \theta}{\int_{0}^{2 \pi}[1+b(\theta)] e^{-a(\theta)} d \theta}+\frac{2 \eta}{\alpha}+\frac{\gamma}{2 \alpha} \sin 2 \theta_{0}, \\
& F\left(\alpha, \eta, \gamma, \theta_{0}\right)=\frac{\int_{0}^{2 \pi} \sin 2\left(\theta-\theta_{0}\right)[1+b(\theta)] e^{-a(\theta)} d \theta}{\int_{0}^{2 \pi}[1+b(\theta)] e^{-a(\theta)} d \theta}+\frac{\gamma}{2 \alpha} \cos 2 \theta_{0},
\end{aligned}
$$

for the terms obtained in (1.10) and (1.11). We will directly calculate (2.17) and (2.18) for given $\alpha, \gamma, \eta, \theta_{0}$. Then we take the contour line of value 0 for $B$ and $F$. The intersection points are what we want to find. The following figures are the contour lines of value 0 of $B$ (continuous line) and $F$ (dashed line) in (figures 2.1-2.6).

From figures (2.1-2.6), we can see that there are multiple points of the contour lines of value 0 of $B$ and $F$ of (2.17) and (2.18), respectively. In the following we give 


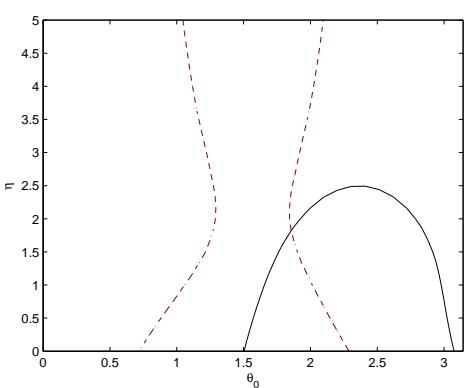

FIG. 2.1. $\alpha=5.0$ and $\gamma=3.0$

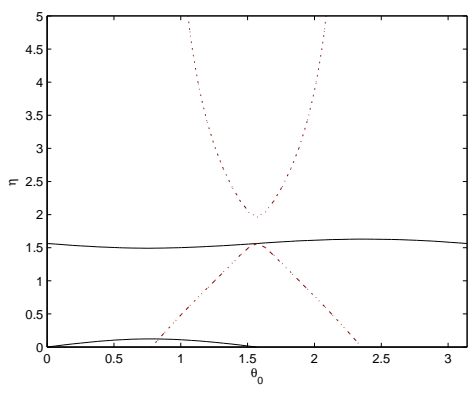

FIG. 2.3. $\alpha=5.1175$ and $\gamma=0.1$

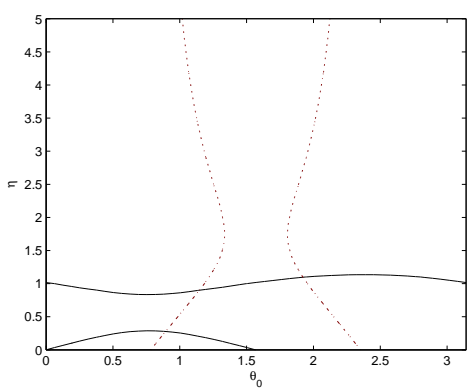

FIG. 2.5. $\alpha=4.5$ and $\gamma=0.1$

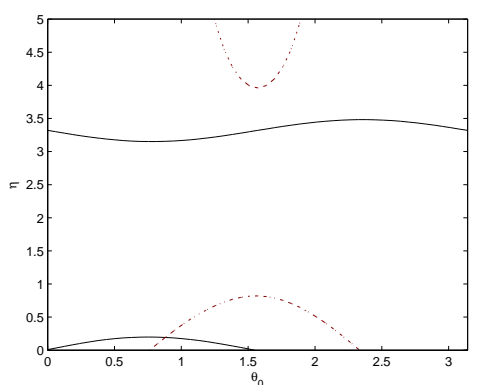

FIG. 2.2. $\alpha=8.0$ and $\gamma=0.5$

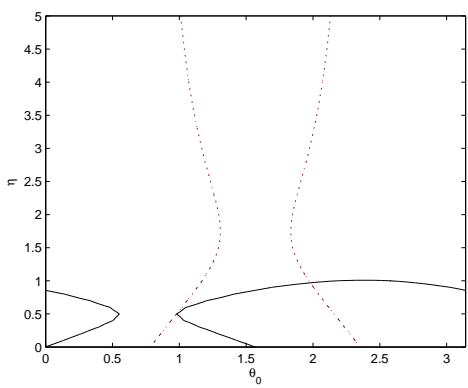

FIG. 2.4. $\alpha=4.3615$ and $\gamma=0.1$

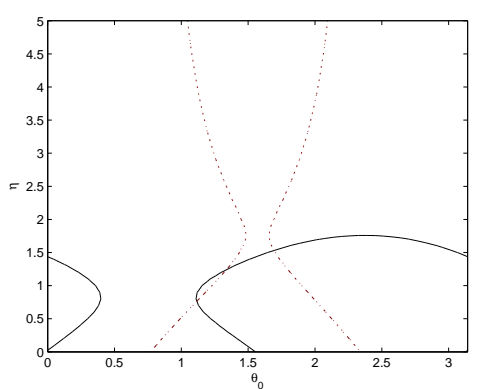

FIG. 2.6. $\alpha=5.0$ and $\gamma=0.5$

\begin{tabular}{|c|c|c|c|c|c|c|c|c|c|c|}
\hline$\gamma$ & 0.01 & 0.01 & 0.01 & 0.15 & 0.15 & 0.15 & 0.17 & 0.17 & 0.2 & 0.3 \\
\hline$\eta$ & 0.133 & 0.97 & 1.06 & 0.47 & 0.78 & 1.12 & 0.60 & 1.13 & 1.15 & 1.19 \\
\hline$\theta_{0}$ & 0.84 & 1.73 & 1.94 & 0.97 & 1.10 & 1.91 & 1.03 & 1.91 & 1.90 & 1.90 \\
\hline$E n$ & -0.70 & -0.72 & -0.72 & -0.59 & -0.59 & -0.63 & -0.57 & -0.62 & -0.60 & -0.53 \\
\hline
\end{tabular}

TABLE 2.1. $\alpha=4.5$, En=energy 


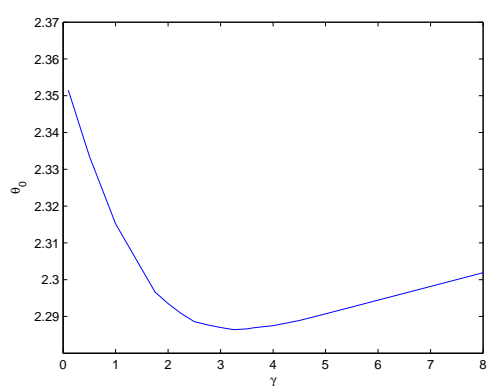

FIG. 2.7. $\alpha=1.0$

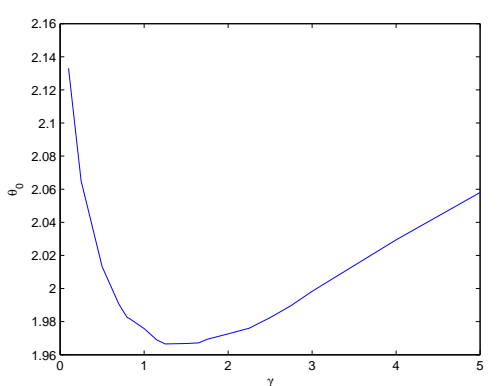

FIG. 2.9. $\alpha=4.0$

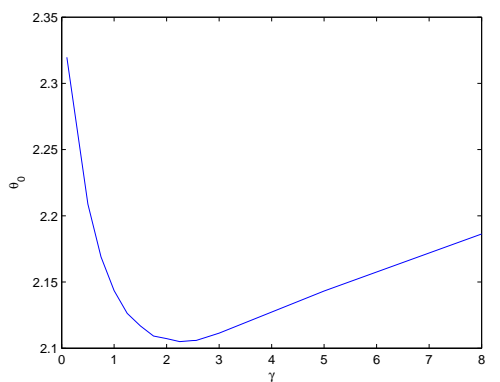

FIG. 2.8. $\alpha=3.0$

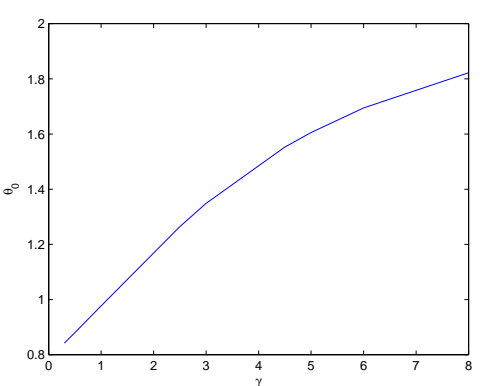

FIG. 2.10. $\alpha=8.0$

\begin{tabular}{|c|c|c|c|c|c|c|c|c|c|c|}
\hline$\gamma$ & 0.4 & 0.5 & 0.75 & 1.0 & 1.5 & 2.0 & 3.0 & 4.0 & 4.5 & 5.0 \\
\hline$\eta$ & 1.23 & 1.27 & 1.33 & 1.38 & 1.47 & 1.59 & 1.73 & 1.93 & 2.02 & 2.03 \\
\hline$\theta_{0}$ & 1.88 & 1.87 & 1.86 & 1.86 & 1.87 & 1.89 & 1.93 & 1.98 & 1.99 & 2.06 \\
\hline
\end{tabular}

TABLE 2.2. $\alpha=4.5$

\begin{tabular}{|c|c|c|c|c|c|c|c|c|c|}
\hline$\gamma$ & 0.05 & 0.05 & 0.05 & 0.1 & 0.1 & 0.1 & 0.4 & 0.4 & 0.4 \\
\hline$\eta$ & 0.07 & 1.46 & 1.48 & 0.14 & 1.45 & 1.49 & 0.57 & 1.33 & 1.52 \\
\hline$\theta_{0}$ & 0.81 & 1.44 & 1.70 & 0.84 & 1.43 & 1.70 & 1.03 & 1.38 & 1.69 \\
\hline energy & -0.55 & -0.59 & -0.60 & -0.51 & -0.55 & -0.56 & -0.25 & -0.28 & -0.34 \\
\hline
\end{tabular}

TABLE 2.3. $\alpha=5.0$

\begin{tabular}{|c|c|c|c|c|c|c|c|c|c|}
\hline$\gamma$ & 0.5 & 0.5 & 0.5 & 0.57 & 0.6 & 0.8 & 1.0 & 1.5 & 2.0 \\
\hline$\eta$ & 0.77 & 1.23 & 1.53 & 1.54 & 1.54 & 1.56 & 1.57 & 1.62 & 1.68 \\
\hline$\theta_{0}$ & 1.12 & 1.34 & 1.70 & 1.70 & 1.70 & 1.71 & 1.73 & 1.75 & 1.79 \\
\hline energy & -0.16 & -0.18 & -0.27 & -0.22 & -0.19 & -0.14 & -0.11 & 0.48 & 0.86 \\
\hline
\end{tabular}

TABLE 2.4. $\alpha=5.0$ 


\begin{tabular}{|c|c|c|c|c|c|c|c|c|c|c|}
\hline$\gamma$ & 2.5 & 3.0 & 3.5 & 4.0 & 4.5 & 5.0 & 6.0 & 7.0 & 8.0 & 10.0 \\
\hline$\eta$ & 1.75 & 1.82 & 1.92 & 2.00 & 2.10 & 2.21 & 2.42 & 2.64 & 2.86 & 3.32 \\
\hline$\theta_{0}$ & 1.75 & 1.86 & 1.89 & 1.91 & 1.94 & 1.96 & 2.00 & 2.03 & 2.06 & 2.10 \\
\hline
\end{tabular}

TABLE 2.5. $\alpha=5.0$

\begin{tabular}{|c|c|c|c|c|c|c|c|c|c|}
\hline$\gamma$ & 0.3 & 0.5 & 1.0 & 3.0 & 5.0 & 8.0 & 10.0 & 12.0 & 15.0 \\
\hline$\eta$ & 0.12 & 0.19 & 0.38 & 1.08 & 1.80 & 2.85 & 3.44 & 3.99 & 4.76 \\
\hline$\theta_{0}$ & 0.84 & 0.88 & 0.98 & 1.35 & 1.61 & 1.82 & 1.91 & 1.97 & 2.03 \\
\hline
\end{tabular}

TABLE 2.6. $\alpha=8.0$

\begin{tabular}{|c|c|c|c|c|c|c|c|c|c|}
\hline$\gamma$ & 0.5 & 0.8 & 1.0 & 3.0 & 5.0 & 8.0 & 10.0 & 12.0 & 15.0 \\
\hline$\eta$ & 0.14 & 0.22 & 0.28 & 0.81 & 1.39 & 2.38 & 3.06 & 3.71 & 4.63 \\
\hline$\theta_{0}$ & 0.86 & 0.90 & 0.930 & 1.23 & 1.47 & 1.70 & 1.79 & 1.86 & 1.94 \\
\hline
\end{tabular}

TABLE 2.7. $\alpha=10.0$.

some tables, which are zeros of $B$ and $F$ for different $\alpha$ and $\gamma$. These tables show the following facts:

(a) When $0<\alpha<\alpha_{1}\left(\alpha_{1} \approx 4.083\right)$, there is only one pair of $\left(\eta, \theta_{0}\right)$ such that $B=0$ and $F=0$ (figure 2.1). Moreover, for $\alpha \in] 0, \alpha_{1}$ [ fixed, the solution $\left(\eta, \theta_{0}\right)$ depends on the shear rate $\gamma$ and $\theta_{0}$ is not a monotone function of $\gamma$. There is a critical point of $\gamma$ such that $\theta_{0}$ attains the minimum. For example, $\gamma=3.5$ is the critical point for $\alpha=1.0$ (figure 2.7). The values $\gamma=2.5,1.5$ are also the critical points for $\alpha=3.0,4.0$ (figures $2.8,2.9$ ), respectively, while the solution $\eta$ is an increasing function of $\gamma$ for $\alpha$ fixed. In fact from the form (2.6) of $a(\theta)$ there is another pair of zeros, denoted by $\left(\eta^{*}, \theta_{0}^{*}\right)$, then $\eta^{*}=-\eta$ and $\theta_{0}^{*}=\theta_{0}+\pi / 2$.

(b) When $\alpha_{1}<\alpha<\alpha_{2}\left(\alpha_{2} \approx 5.125\right)$, there are multiple pairs of solutions $\left(\eta, \theta_{0}\right)$ such that $B=0$ and $F=0$ (ref. Tables 2.1-2.4). The number of solutions depends on the choice of $\gamma$. For example, we can see that $\left(\eta, \theta_{0}\right)=(0.1333,0.8374)$, $(0.967,1.728),(1.0596,1.935)$ are solutions of $B=0$ and $F=0$ for $\gamma=0.01$ and $\alpha=4.5$ from Table 2.2. When $\alpha=4.5$ and $\gamma=0.1685$, there are two solutions $\left(\eta, \theta_{0}\right)=$ $(0.6,1.025),(1.1346,1.9092)$. But when $\alpha=4.5$ and $\gamma>0.6$, there is only one solution. Moreover, comparing Table 2.4 and Table 2.2, we can see that $\gamma$ depends on $\alpha \in] \alpha_{1}, \alpha_{2}$ [, for which the equations $B=0$ and $F=0$ has multiple solutions. But not all these solutions are stable. In section 4 we will investigate the stability of these solutions.

(c) When $\alpha>\alpha_{2}$, there is only a pair of solution $\left(\eta, \theta_{0}\right)$ which satisfies $B=0$ and $F=0$ (figure 2.2). Moreover, for $\alpha \in] \alpha_{2}, \infty\left[\right.$ fixed, $\theta_{0}$ and $\eta$ are increasing functions of the shear rate $\gamma$, e.g., from (figure 2.10) we can see the profile of $\theta_{0}(\gamma)$ for $\alpha=8.0$. 


\section{Stability of multiple solutions}

In this section we will investigate the stability of the multiple solutions with respect to the energy functional. We use (1.6) and (2.11) to obtain

$$
\begin{aligned}
E(\psi) & =\int_{0}^{2 \pi}\left[\psi \ln \psi+\frac{1}{2} \psi a(\theta)\right] d \theta \\
& =\int_{0}^{2 \pi} \psi[\ln (1+b(\theta))-a(\theta)-\ln Z]+\frac{1}{2} \psi\left[\frac{\alpha}{2}+\eta \cos 2\left(\theta-\theta_{0}\right)+\frac{\gamma \theta}{2}\right] d \theta .
\end{aligned}
$$

Then (2.6) gives

$$
\begin{aligned}
E(\psi)= & \int_{0}^{2 \pi}\left\{\psi\left[\ln (1+b(\theta))-\left(\frac{\alpha}{2}+\eta \cos 2\left(\theta-\theta_{0}\right)+\frac{\gamma \theta}{2}\right)-\ln Z\right]\right. \\
& \left.+\frac{1}{2} \psi\left[\frac{\alpha}{2}+\eta \cos 2\left(\theta-\theta_{0}\right)+\frac{\gamma \theta}{2}\right]\right\} d \theta \\
= & \int_{0}^{2 \pi} \psi\left\{\ln (1+b(\theta))-\frac{1}{2}\left[\frac{\alpha}{2}+\eta \cos 2\left(\theta-\theta_{0}\right)+\gamma \theta-\frac{\gamma}{4} \sin 2 \theta\right]-\ln Z\right\} d \theta \\
= & \frac{1}{Z} \int_{0}^{2 \pi}[1+b(\theta)] e^{-a(\theta)}\left[\ln (1+b(\theta))-\frac{\eta}{2} \cos 2\left(\theta-\theta_{0}\right)-\frac{\gamma \theta}{4}\right] d \theta \\
& -\frac{\alpha}{4}-\ln Z
\end{aligned}
$$

Thus, we can calculate the energy functional for $\eta, \theta_{0}$ determined by (1.10) and (1.11) as $\alpha, \gamma$ given. For example, from Table 2.1, we can see that the energies of points $\left(\eta, \theta_{0}\right)=(0.967,1.728),(1.0596,1.935)$ are almost -0.72 and less than the energy of other solution as $\alpha=4.5$ and $\gamma=0.01$. So these two points are stable points. Here we show the Table 2.3 and 2.4 to display the energy for the multiple solutions of (1.10) and (1.11) for $\alpha=4.5,5.0$, respectively, and $\gamma$ given. The points with low values are the stable points.

\section{Conclusion}

In this work we have given the explicit expression of the equilibrium to the $1 \mathrm{D}$ Doi model in the shear flow. The difference from this expression to the MaxwellBoltzmann distribution without flow can be seen. Here are two parameters, the interaction intensity among particles $\alpha$ and the shear rate $\gamma$, to determine the states of the particles in the general one dimension shear flow of polymeric fluids. According to our discussion, we know that the equilibrium of these particles depends on the competition between $\alpha$ and $\gamma$. When the interaction intensity $\alpha$ is less than the first critical point $\alpha_{1} \approx 4.083$ or larger than the second critical point $\alpha_{2} \approx 5.125$, the steady state of these molecules is uniquely determined by $\alpha$ and $\gamma$, where $\gamma$ can be arbitrary. But when $\alpha \in] \alpha_{1}, \alpha_{2}$ [, it is another story. There are multiple steady states of these particles. It is possible to have one, two, or three equilibria. This is completely dependent on the shear rate. When the shear rate is very large, the equilibrium is also uniquely determined. While the shear rate is not large enough, the equilibrium will be multiple steady states. However, not all equilibria are stable. From the discussion of the energy functional, we can see that it is possible that there are one or two stable equilibria for some $\alpha$ and $\gamma$.

Acknowledgement. The authors are grateful for the referee's suggestions. 


\section{REFERENCES}

[1] P. Constantin, I. Kevrekidis and E.S. Titi, Asymptotic states of a Smoluchowski equation, Arch. Rat. Mech. Anal., 174, 365-384, 2004.

[2] P. Constantin, I. Kevrekidis and E.S. Titi, Remarks on a Smoluchowski equation, Discrete and Continuous Dynamical Systems, 11, 101-112, 2004.

[3] P. Constantin and J. Vukadinovic, Note on the number of steady states for a $2 D$ Smoluchowski equation, Nonlinearity, 18, 441-443, 2005.

4] M. Doi and S.F. Edwards, The Theory of Polymer Dynamics, Oxford University Press, 1986.

[5] P.G. de Gennes and J. Prost, The Physics of Liquid Crystals, 2nd ed., Oxford Science Publications, 1993

[6] M. Doi and R.G. Larson, The effect of steady flow fields on the isotropic-nematic phase transition of rigid rod-like polymers, J. Chem. Phy., 92, 792-800, 1990.

[7] I. Fatkullin and V. Slastikov, A note on the Onsager model of nematic phase transitions, Commun. Math. Sci., 3(1), 21-26, 2005.

[8] I. Fatkullin and V. Slastikov, Critical points of the Onsager functional on a sphere, Nonlinearity, $18,2565-2580,2005$.

[9] V. Faraoni, M. Grosso, S. Crescitelli and P.L. Maffettone, The rigid-rod model for nematic polymers: an analysis of the shear flow problem, J. Rheol., 43, 829-843, 1999.

[10] M.G. Forest, R. Zhou and Q. Wang, Full-tensor alignment criteria for sheared nematic polymers, J. Rheol., 47, 105-127, 2003.

[11] M.G. Forest, Q. Wang and R. Zhou, The weak shear kinetic phase diagram for nematic polymers, Rheological Acta, 43, 17-37, 2004.

[12] G.H. Ji, Q. Wang, P.W. Zhang, H.Y Wang and H. Zhou, Steady states and their stability of homogeneous, rigid extended nematic polymers under imposed magnetic fields, Commun. Math. Sci., 5, 917-950, 2007.

[13] G.H. Ji, Q. Wang, P.W. Zhang and H. Zhou, Study of phase transition in homogeneous, rigid extended nematics and magnetic suspensions using an order-reduction method, Phy. Fluids, 18, 123103, 2006.

[14] R. Larson and H. Ottinger, Effect of molecular elasticity on out-of-plane orientations in shearing flows of liquid-crystalline polymers, Macromolecules, 24, 6270-6282, 1991.

[15] J.H. Lee, M.G. Forest and R.H. Zhou, Aligenment and rheo-oscillator criteria for sheared nematic polymer films in the monolayer limit, Discrete and Continuos Dynamical Systems B, 6, 339-356, 2006.

[16] C. Luo, H. Zhang and P.W. Zhang, The structure of equilibrium solutions of the onedimensional Doi equation, Nonlinearity, 18, 379-389, 2005.

[17] H.L. Liu, H. Zhang and P.W. Zhang, Axial symmstry and classification of stationary solutions of Doi-Onsager equation on the sphere with Maier-Saupe potential, Commun. Math. Sci., 3(2), 201-218, 2005.

[18] H.L. Liu, Global orientation dynamics for liquid crystalline polymers, Physica D, 228, 122-129, 2007.

[19] G. Marrucci and P.L. Maffettone, Description of the liquid crystalline phase of rodlike polymers at high shear rates, Macromolecules, 22, 4076-4082, 1989.

[20] G. Marrucci and P.L. Maffettone, Bifurcation analysis of a molecular model for nematic polymers in the shear flow, J. Non-Newtonian Fluid Mech., 59, 73-91, 1995.

[21] W. Maier and A. Saupe, A simple molecular statistical theory of the nematic crystalline-liquid phase, I.Z. Naturforsch 14 A, 882-889, 1959.

[22] L. Onsager, The effects of shape on the interaction of colloidal particles, Ann. N.Y. Acad. Sci., 51, 627-659, 1949.

[23] H. Zhou, H. Wang, G. Forest and Q. Wang, A new proof on uniaxial equilibria of a three dimensional Smoluchowski equation, Nonlinearity, 18, 2815-2825, 2005.

[24] H. Zhang, and P.W. Zhang, Stable dynamic states at the nematic liquid crystals in weak shear flow, Physica D, 232, 156-165, 2007.

[25] H. Zhang and P.W. Zhang, Review on Doi-Onsager model in polymeric fluids, Wenbin Liu, Michael Ng and Zhong-Ci Shi (eds), Recent Progress in Scientific Computing, 155-167, 2007.

[26] H. Zhang and P.W. Zhang, On the new multiscale rodlike model of polymeric fluids, SIAM J. Math. Anal., 40, 1246-1271, 2008. 